\title{
Metric Dimension in fuzzy(neutrosophic) Graphs-VII
}

\author{
Henry Garrett \\ Independent Researcher \\ DrHenryGarrett@gmail.com \\ Twitter's ID: @DrHenryGarrett | @DDrHenryGarrett.wordpress.com
}

\begin{abstract}
New notion of dimension as set, as two optimal numbers including metric number, dimension number and as optimal set are introduced in individual framework and in formation of family. Behaviors of twin and antipodal are explored in fuzzy(neutrosophic) graphs. Fuzzy(neutrosophic) graphs, under conditions, fixed-edges, fixed-vertex and strong fixed-vertex are studied. Some classes as path, cycle, complete, strong, t-partite, bipartite, star and wheel in the formation of individual case and in the case, they form a family are studied in the term of dimension. Fuzzification (neutrosofication) of twin vertices but using crisp concept of antipodal vertices are another approaches of this study. Thus defining two notions concerning vertices which one of them is fuzzy(neutrosophic) titled twin and another is crisp titled antipodal to study the behaviors of cycles which are partitioned into even and odd, are concluded. Classes of cycles according to antipodal vertices are divided into two classes as even and odd. Parity of the number of edges in cycle causes to have two subsections under the section is entitled to antipodal vertices. In this study, the term dimension is introduced on fuzzy(neutrosophic) graphs. The locations of objects by a set of some junctions which have distinct distance from any couple of objects out of the set, are determined. Thus it's possible to have the locations of objects outside of this set by assigning partial number to any objects. The classes of these specific graphs are chosen to obtain some results based on dimension. The types of crisp notions and fuzzy(neutrosophic) notions are used to make sense about the material of this study and the outline of this study uses some new notions which are crisp and fuzzy(neutrosophic). Some questions and problems are posed concerning ways to do further studies on this topic. Basic familiarities with fuzzy(neutrosophic) graph theory and graph theory are proposed for this article.
\end{abstract}

Keywords: Fuzzy Graphs, Neutrosophic Graphs, Dimension AMS Subject Classification: 05C17, 05C22, 05E 45

\section{Background}

Fuzzy set in Ref. [15], neutrosophic set in Ref. [2], related definitions of other sets in Refs. [2,13,14], graphs and new notions on them in Refs. [5-11], neutrosophic graphs in Ref. [3], studies on neutrosophic graphs in Ref. [1], relevant definitions of other graphs based on fuzzy graphs in Ref. [12], related definitions of other graphs based on neutrosophic graphs in Ref. [4], are proposed. 
In this section, I use two subsections to illustrate a perspective about the background of this study.

\subsection{Motivation and Contributions}

In this study, there's an idea which could be considered as a motivation.

Question 1.1. Is it possible to use mixed versions of ideas concerning "crisp", "fuzzy" and "neutrosophic" to define some notions which are applied to fuzzy(neutrosophic) graphs?

It's motivation to find notions to use in any classes of fuzzy(neutrosophic) graphs. Real-world applications about locating the item, are another thoughts which lead to be considered as motivation. Distance and path amid two items have key roles to locate. Thus they're used to define new ideas which conclude to the structure of metric dimension. The concept of connectedness inspire to study the behavior of path and distance in the way that, both individual fuzzy(neutrosophic) graphs and family of them are the cases of study.

The framework of this study is as follows. In section (2), I introduce main definitions alongside some examples to clarify about them. In section (3), general attributes are given. In section (4), one idea about specific fuzzy(neutrosophic) vertices is used to form the results for fuzzy(neutrosophic) graphs and family of them. In section (5), one idea about specific crisp vertices is used to form the results for fuzzy(neutrosophic) graphs and family of them especially fuzzy(neutrosophic) cycles as two subsections, In section (6), the results are extended for fuzzy(neutrosophic) graphs and family of them. In section (7), two applications are posed for fuzzy(neutrosophic) graphs and family of them. In section (8), some problems and questions for further studies are proposed. In section (9), gentle discussion about results and applications are featured. In section (10), a brief overview concerning advantages and limitations of this study alongside conclusions are formed.

\subsection{Preliminaries}

To clarify about the models, I use some definitions and results, and in this way, results have a key role to make sense about the definitions and to introduce new ways to use on these models in the terms of new notions. For instance, the concept of complete is used to specialize a graph in every environment. To differentiate, I use an adjective or prefix in every definition. Two adjectives "fuzzy" and "neutrosophic" are used to distinguish every graph or classes of graph or any notion on them.

$G:(V, E)$ is called a crisp graph where $V$ is a set of objects and $E$ is a subset of $V \times V$ such that this subset is symmetric. A crisp graph $G:(V, E)$ is called a fuzzy graph $G:(\sigma, \mu)$ where $\sigma: V \rightarrow[0,1]$ and $\mu: E \rightarrow[0,1]$ such that $\mu(x y) \leq \sigma(x) \wedge \sigma(y)$ for all $x y \in E$. A crisp graph $G:(V, E)$ is called a neutrosophic graph $G:(\sigma, \mu)$ where $\sigma=\left(\sigma_{1}, \sigma_{2}, \sigma_{3}\right): V \rightarrow[0,1]$ and $\mu=\left(\mu_{1}, \mu_{2}, \mu_{3}\right): E \rightarrow[0,1]$ such that $\mu(x y) \leq \sigma(x) \wedge \sigma(y)$ for all $x y \in E$. A crisp graph $G:(V, E)$ is called a crisp complete where $\forall x \in V, \forall y \in V, x y \in E$. A fuzzy graph $G:(\sigma, \mu)$ is called fuzzy complete where it's complete and $\mu(x y)=\sigma(x) \wedge \sigma(y)$ for all $x y \in E$. A neutrosophic graph $G:(\sigma, \mu)$ is called a neutrosophic complete where it's complete and $\mu(x y)=\sigma(x) \wedge \sigma(y)$ for all $x y \in E$. An $N$ which is a set of vertices, is called fuzzy(neutrosophic) cardinality and it's denoted by $|N|$ such that $|N|=\Sigma_{n \in N} \sigma(n)$. A crisp graph $G:(V, E)$ is called a crisp strong. A fuzzy graph $G:(\sigma, \mu)$ is called fuzzy strong where $\mu(x y)=\sigma(x) \wedge \sigma(y)$ for all $x y \in E$. A neutrosophic graph $G:(\sigma, \mu)$ is called a neutrosophic strong where $\mu(x y)=\sigma(x) \wedge \sigma(y)$ for all $x y \in E$. A distinct sequence of vertices $v_{0}, v_{1}, \cdots, v_{n}$ in a 
crisp graph $G:(V, E)$ is called crisp path with length $n$ from $v_{0}$ to $v_{n}$ where $v_{i} v_{i+1} \in E, i=0,1, \cdots, n-1$. If one edge is incident to a vertex, the vertex is called leaf. A path $v_{0}, v_{1}, \cdots, v_{n}$ is called fuzzy path where $\mu\left(v_{i} v_{i+1}\right)>0, i=0,1, \cdots, n-1$. A path $v_{0}, v_{1}, \cdots, v_{n}$ is called neutrosophic path where $\mu\left(v_{i} v_{i+1}\right)>0, i=0,1, \cdots, n-1$. Let $P: v_{0}, v_{1}, \cdots, v_{n}$ be fuzzy(neutrosophic) path from $v_{0}$ to $v_{n}$ such that it has minimum number of vertices as possible, then $d\left(v_{0}, v_{n}\right)$ is defined as $\sum_{i=0}^{n} \mu\left(v_{i-1} v_{i}\right)$. A path $v_{0}, v_{1}, \cdots, v_{n}$ with exception of $v_{0}$ and $v_{n}$ in a crisp graph $G:(V, E)$ is called crisp cycle with length $n$ for $v_{0}$ where $v_{0}=v_{n}$. A cycle $v_{0}, v_{1}, \cdots, v_{0}$ is called fuzzy cycle where there are two edges $x y$ and $u v$ such that $\mu(x y)=\mu(u v)=\bigwedge_{i=0,1, \cdots, n-1} \mu\left(v_{i} v_{i+1}\right)$. A cycle $v_{0}, v_{1}, \cdots, v_{0}$ is called neutrosophic cycle where there are two edges $x y$ and $u v$ such that

$\mu(x y)=\mu(u v)=\bigwedge_{i=0,1, \cdots, n-1} \mu\left(v_{i} v_{i+1}\right)$. A fuzzy(neutrosophic) cycle is called odd if the number of its vertices is odd. Similarly, a fuzzy(neutrosophic) cycle is called even if the number of its vertices is even. A fuzzy(neutrosophic) graph is called

fuzzy(neutrosophic) t-partite if $V$ is partitioned to $t$ parts, $V_{1}, V_{2}, \cdots, V_{t}$ and the edge $x y$ implies $x \in V_{i}$ and $y \in V_{j}$ where $i \neq j$. If it's fuzzy(neutrosophic) complete, then it's denoted by $K_{\sigma_{1}, \sigma_{2}, \cdots, \sigma_{t}}$ where $\sigma_{i}$ is $\sigma$ on $V_{i}$ instead $V$ which mean $x \notin V_{i}$ induces $\sigma_{i}(x)=0$. If $t=2$, then it's called fuzzy(neutrosophic) complete bipartite and it's denoted by $K_{\sigma_{1}, \sigma_{2}}$ especially, if $\left|V_{1}\right|=1$, then it's called fuzzy (neutrosophic) star and it's denoted by $S_{1, \sigma_{2}}$. In this case, the vertex in $V_{1}$ is called center and if a vertex joins to all vertices of fuzzy(neutrosophic), it's called fuzzy(neutrosophic) wheel and it's denoted by $W_{1, \sigma_{2}}$. A set is $\mathbf{n}$-set if its

Table 1. Crisp-fying, Fuzzy-fying and Neutrosophic-fying

\begin{tabular}{|c|c|c|}
\hline Crisp Graphs & Fuzzy Graphs & Neutrosophic Graphs \\
\hline Crisp Complete & Fuzzy Complete & Neutrosophic Complete \\
\hline Crisp Strong & Fuzzy Strong & Neutrosophic Strong \\
\hline Crisp Path & Fuzzy Path & Neutrosophic Path \\
\hline Crisp Cycle & Fuzzy Cycle & Neutrosophic Cycle \\
\hline Crisp t-partite & Fuzzy t-partite & Neutrosophic t-partite \\
\hline Crisp Bipartite & Fuzzy Bipartite & Neutrosophic Bipartite \\
\hline Crisp Star & Fuzzy Star & Neutrosophic Star \\
\hline Crisp Wheel & Fuzzy Wheel & Neutrosophic Wheel \\
\hline
\end{tabular}

cardinality is $n$. A fuzzy vertex set is the subset of vertex set of (neutrosophic) fuzzy graph such that the values of these vertices are considered. A fuzzy edge set is the subset of edge set of (neutrosophic) fuzzy graph such that the values of these edges are considered. Let $\mathcal{G}$ be a family of fuzzy graphs or neutrosophic graphs. This family have fuzzy(neutrosophic) common vertex set if all graphs have same vertex set and its values but edges set is subset of fuzzy edge set. A (neutrosophic) fuzzy graph is called fixed-edge fuzzy(neutrosophic) graph if all edges have same values. A (neutrosophic) fuzzy graph is called fixed-vertex fuzzy(neutrosophic) graph if all vertices have same values. A couple of vertices $x$ and $y$ is called crisp twin vertices if either $N(x)=N(y)$ or $N[x]=N[y]$ where $\forall x \in V, N(x)=\{y \mid x y \in E\}, N[x]=N(x) \cup\{x\}$. Two vertices $t$ and $t^{\prime}$ are called fuzzy (neutrosophic) twin vertices if $N(t)=N\left(t^{\prime}\right)$ and $\mu(t s)=\mu\left(t^{\prime} s\right)$, for all $s \in N(t)=N\left(t^{\prime}\right) \cdot \max _{x, y \in V(G)}|E(P(x, y))|$ is called diameter of $G$ and it's denoted by $D(G)$ where $|E(P(x, y))|$ is the number of edges on the path from $x$ to $y$. For any given vertex $x$ if there's exactly one vertex $y$ such that $\min _{P(x, y)}|E(P(x, y))|=D(G)$, then a couple of vertices $x$ and $y$ are called antipodal vertices. 
Table 2. Crisp-fying, Fuzzy-fying and Neutrosophic-fying

\begin{tabular}{l|lcc|}
\hline & Crisp Vertex Set & Fuzzy Vertex Set & Neutrosophic Vertex Set \\
\hline & Crisp Edge Set & Fuzzy Edge Set & Neutrosophic Edge Set \\
Crisp Common & Fuzzy Common & Neutrosophic Common & \\
Crisp Fixed-edge & Fuzzy Fixed-edge & Neutrosophic Fixed-edge & \\
Crisp Fixed-vertex & Fuzzy Fixed-vertex & Neutrosophic Fixed-vertex & \\
Crisp Twin & Fuzzy Twin & Neutrosophic Twin & \\
\hline
\end{tabular}

\section{Definitions}

I use the notion of vertex in fuzzy(neutrosophic) graphs to define new notions which state the relation amid vertices. In this way, the set of vertices are distinguished by another set of vertices.

Definition 2.1. Let $G=(V, \sigma, \mu)$ be a fuzzy(neutrosophic) graph. A vertex $m$ fuzzy(neutrosophic)-resolves vertices $f_{1}$ and $f_{2}$ if $d\left(m, f_{1}\right) \neq d\left(m, f_{2}\right)$. A set $M$ is fuzzy(neutrosophic)-resolving set if for every couple of vertices $f_{1}, f_{2} \in V \backslash M$, there's a vertex $m \in M$ such that $m$ fuzzy(neutrosophic)-resolves $f_{1}$ and $f_{2} .|M|$ is called fuzzy(neutrosophic)-metric number of $G$ and

$$
\underset{S \text { is fuzzy(neutrosophic)-resolving set }}{\min } \Sigma_{s \in S} \sigma(s)=\Sigma_{m \in M} \sigma(m)
$$

is called fuzzy(neutrosophic)-metric dimension of $G$ and if

$$
\min _{S \text { is fuzzy(neutrosophic)-resolving set }} \Sigma_{s \in S} \sigma(s)=\Sigma_{m \in M} \sigma(m)
$$

where $M$ is fuzzy(neutrosophic)-resolving set, then $M$ is called fuzzy(neutrosophic)-metric set of $G$.

Example 2.2. Let $G$ be a fuzzy(neutrosophic) graph as figure (1). By applying Table (3), the 1-set is explored which its cardinality is minimum. $\left\{f_{6}\right\}$ and $\left\{f_{4}\right\}$ are 1-set which has minimum cardinality amid all sets of vertices but $\left\{f_{4}\right\}$ isn't fuzzy(neutrosophic)-resolving set and $\left\{f_{6}\right\}$ is fuzzy(neutrosophic)-resolving set. Thus there's no fuzzy(neutrosophic)-metric set but $\left\{f_{6}\right\}$. $f_{6}$ fuzzy(neutrosophic)-resolves all given couple of vertices. Therefore one is fuzzy(neutrosophic)-metric number of $G$ and 0.13 is fuzzy(neutrosophic)-metric dimension of $G$. By using Table (3), $f_{4}$ doesn't fuzzy(neutrosophic)-resolve $f_{2}$ and $f_{6} . f_{4}$ doesn't fuzzy(neutrosophic)-resolve $f_{1}$ and $f_{5}$, too.

Table 3. Distances of Vertices from sets of vertices $\left\{f_{6}\right\}$ and $\left\{f_{4}\right\}$ in fuzzy(neutrosophic) Graph $G$.

\begin{tabular}{c|lccccc}
\hline Vertices & $f_{1}$ & $f_{2}$ & $f_{3}$ & $f_{4}$ & $f_{5}$ & $f_{6}$ \\
\hline$f_{6}$ & 0.22 & 0.26 & 0.39 & 0.24 & 0.13 & 0 \\
Vertices & $f_{1}$ & $f_{2}$ & $f_{3}$ & $f_{4}$ & $f_{5}$ & $f_{6}$ \\
$f_{4}$ & 0.11 & 0.24 & 0.37 & 0 & 0.11 & 0.24 \\
\hline
\end{tabular}

Definition 2.3. Consider $\mathcal{G}$ as a family of fuzzy(neutrosophic) graphs on a common vertex set $V$. A vertex $m$ simultaneously fuzzy(neutrosophic)-resolves vertices $f_{1}$ and $f_{2}$ if $d_{G}\left(m, f_{1}\right) \neq d_{G}\left(m, f_{2}\right)$, for all $G \in \mathcal{G}$. A set $M$ is simultaneously

fuzzy(neutrosophic)-resolving set if for every couple of vertices $f_{1}, f_{2} \in V \backslash M$, there's a 


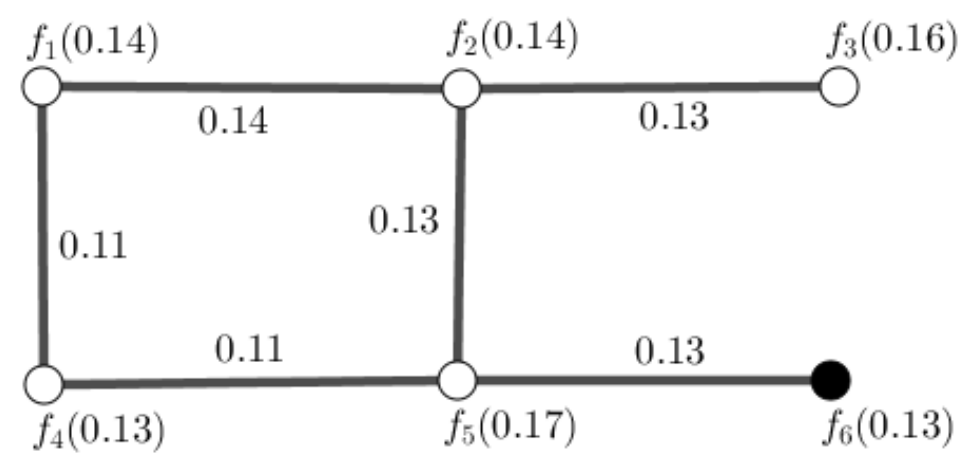

Figure 1. Black vertex $\left\{f_{6}\right\}$ is only fuzzy(neutrosophic)-metric set amid all sets of vertices for fuzzy(neutrosophic) graph $G$.

vertex $m \in M$ such that $m$ resolves $f_{1}$ and $f_{2}$, for all $G \in \mathcal{G} .|M|$ is called simultaneously fuzzy(neutrosophic)-metric number of $\mathcal{G}$ and

$$
\min _{S \text { is fuzzy(neutrosophic)-resolving set }} \Sigma_{s \in S} \sigma(s)=\Sigma_{m \in M} \sigma(m)
$$

is called simultaneously fuzzy(neutrosophic)-metric dimension of $\mathcal{G}$ and if

$$
\min _{S \text { is fuzzy(neutrosophic)-resolving set }} \Sigma_{s \in S} \sigma(s)=\Sigma_{m \in M} \sigma(m)
$$

where $M$ is fuzzy(neutrosophic)-resolving set, then $M$ is called simultaneously fuzzy(neutrosophic)-metric set of $\mathcal{G}$.

Example 2.4. Let $\mathcal{G}=\left\{G_{1}, G_{2}, G_{3}\right\}$ be a collection of fuzzy(neutrosophic) graphs with common fuzzy(neutrosophic) vertex set and a subset of fuzzy(neutrosophic) edge set as figure (2). By applying Table (4), the 1-set is explored which its cardinality is minimum. $\left\{f_{2}\right\}$ and $\left\{f_{4}\right\}$ are 1-set which has minimum cardinality amid all sets of vertices. $\left\{f_{4}\right\}$ is as fuzzy(neutrosophic)-resolving set as $\left\{f_{6}\right\}$ is. Thus there's no fuzzy(neutrosophic)-metric set but $\left\{f_{4}\right\}$ and $\left\{f_{6}\right\} . f_{6}$ as fuzzy(neutrosophic)-resolves all given couple of vertices as $f_{4}$. Therefore one is fuzzy(neutrosophic)-metric number of $\mathcal{G}$ and 0.13 is fuzzy(neutrosophic)-metric dimension of $\mathcal{G}$. By using Table (4), $f_{4}$ fuzzy(neutrosophic)-resolves all given couple of vertices.

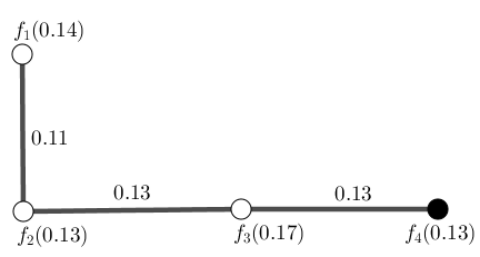

$G_{1}$

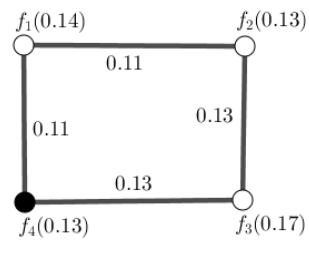

$G_{2}$

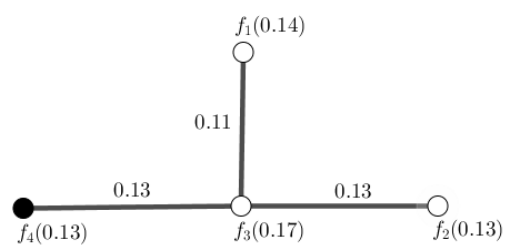

$G_{3}$

Figure 2. Black vertex $\left\{f_{4}\right\}$ and the set of vertices $\left\{f_{2}\right\}$ are simultaneously fuzzy(neutrosophic)-metric set amid all sets of vertices for family of fuzzy(neutrosophic) graphs $\mathcal{G}$.

\section{General Relationships}

Proposition 3.1. Let $G$ be a fuzzy(neutrosophic) path. Then every leaf is fuzzy(neutrosophic)-resolving set. 
Table 4. Distances of Vertices from set of vertices $\left\{f_{6}\right\}$ in Family of fuzzy(neutrosophic) Graphs $\mathcal{G}$.

\begin{tabular}{c|lccc}
\hline Vertices of $G_{1}$ & $f_{1}$ & $f_{2}$ & $f_{3}$ & $f_{4}$ \\
\hline$f_{4}$ & 0.37 & 0.26 & 0.13 & 0 \\
Vertices of $G_{2}$ & $f_{1}$ & $f_{2}$ & $f_{3}$ & $f_{4}$ \\
$f_{4}$ & 0.11 & 0.22 & 0.13 & 0 \\
Vertices of $G_{3}$ & $f_{1}$ & $f_{2}$ & $f_{3}$ & $f_{4}$ \\
$f_{4}$ & 0.24 & 0.26 & 0.13 & 0 \\
\hline
\end{tabular}

Proof. Let $l$ be a leaf. For every given a couple of vertices $f_{i}$ and $f_{j}$, I get $d\left(l, f_{i}\right) \neq d\left(l, f_{j}\right)$. Since if I reassign indexes to vertices such that every vertex $f_{i}$ and $l$ have $i$ vertices amid themselves, then $d\left(l, f_{i}\right)=\Sigma_{j \leq i} \mu\left(f_{j} f_{i}\right) \leq i$. Thus $j \leq i$ implies

$\Sigma_{t \leq j} \mu\left(f_{t} f_{j}\right)+\Sigma_{j \leq s \leq i} \mu\left(f_{s} f_{i}\right)>\Sigma_{j \leq i} \mu\left(f f_{i}\right) \equiv d\left(l, f_{j}\right)+c=d\left(l, f_{i}\right) \equiv d\left(l, f_{j}\right)<d\left(l, f_{i}\right)$.

Therefore, by $d\left(l, f_{j}\right)<d\left(l, f_{i}\right)$, I get $d\left(l, f_{i}\right) \neq d\left(l, f_{j}\right) . f_{i}$ and $f_{j}$ are arbitrary so $l$ fuzzy(neutrosophic)-resolves any given couple of vertices $f_{i}$ and $f_{j}$ which implies $\{l\}$ is a fuzzy(neutrosophic)-resolving set.

Corollary 3.2. Let $G$ be a fixed-edge fuzzy(neutrosophic) path. Then every leaf is fuzzy(neutrosophic)-resolving set.

Proof. Let $l$ be a leaf. For every given couple of vertices, $f_{i}$ and $f_{j}$, I get $d\left(l, f_{i}\right)=c i \neq d\left(l, f_{j}\right)=c j$. It implies $l$ fuzzy(neutrosophic)-resolves any given couple of vertices $f_{i}$ and $f_{j}$ which implies $\{l\}$ is a fuzzy(neutrosophic)-resolving set.

Corollary 3.3. Let $G$ be a fixed-vertex fuzzy(neutrosophic) path. Then every leaf is fuzzy(neutrosophic)-metric set, fuzzy(neutrosophic)-metric number is one and fuzzy(neutrosophic)-metric dimension is c where $c=\sigma(f), f \in V$.

Proof. By Proposition (3.1), every leaf is fuzzy(neutrosophic)-resolving set. By $c=\sigma(f), \forall f \in V$, every leaf is fuzzy(neutrosophic)-metric set,

fuzzy(neutrosophic)-metric number is one and fuzzy(neutrosophic)-metric dimension is c.

Proposition 3.4. Let $G$ be a fuzzy(neutrosophic) path. Then a set including every couple of vertices is fuzzy(neutrosophic)-resolving set.

Proof. Let $f$ and $f^{\prime}$ be a couple of vertices. For every given a couple of vertices $f_{i}$ and $f_{j}$, I get either $d\left(f, f_{i}\right) \neq d\left(f, f_{j}\right)$ or $d\left(f^{\prime}, f_{i}\right) \neq d\left(f^{\prime}, f_{j}\right)$.

Corollary 3.5. Let $G$ be a fixed-edge fuzzy(neutrosophic) path. Then every set containing couple of vertices is fuzzy(neutrosophic)-resolving set.

Proof. Consider $G$ is a fuzzy(neutrosophic) path. Thus by Proposition (3.4), every set containing couple of vertices is fuzzy(neutrosophic)-resolving set. So it holds for any given fixed-edge path fuzzy(neutrosophic) graph.

\section{Fuzzy(Neutrosophic) Twin Vertices}

Proposition 4.1. Let $G$ be a fuzzy(neutrosophic) graph. An $(k-1)$-set from an $k$-set of fuzzy(neutrosophic) twin vertices is subset of a fuzzy(neutrosophic)-resolving set.

Proof. If $t$ and $t^{\prime}$ are fuzzy(neutrosophic) twin vertices, then $N(t)=N\left(t^{\prime}\right)$ and $\mu(t s)=\mu\left(t^{\prime} s\right)$, for all $s \in N(t)=N\left(t^{\prime}\right)$. 
Corollary 4.2. Let $G$ be a fuzzy(neutrosophic) graph. The number of fuzzy(neutrosophic) twin vertices is $n-1$. Then fuzzy(neutrosophic)-metric number is $n-2$.

Proof. Let $f$ and $f^{\prime}$ be two vertices. By supposition, the cardinality of set of fuzzy(neutrosophic) twin vertices is $n-2$. Thus there are two cases. If both are fuzzy(neutrosophic) twin vertices, then $N(f)=N\left(f^{\prime}\right)$ and $\mu(f s)=\mu\left(f^{\prime} s^{\prime}\right), \forall s \in N(f)$, $\forall s^{\prime} \in N\left(f^{\prime}\right)$. It implies $d(f, t)=d(f, t)$ for all $t \in V$. Thus suppose if not, then let $f$ be a vertex which isn't fuzzy(neutrosophic) twin vertices with any given vertex and let $f^{\prime}$ be a vertex which is fuzzy(neutrosophic) twin vertices with any given vertex but not $f$. By supposition, it's possible and this is only case. Therefore, any given distinct vertex fuzzy(neutrosophic)-resolves $f$ and $f^{\prime}$. Then $V \backslash\left\{f, f^{\prime}\right\}$ is fuzzy(neutrosophic)-resolving set. It implies fuzzy(neutrosophic)-metric number is $n-2$.

Corollary 4.3. Let $G$ be a fuzzy(neutrosophic) graph. The number of fuzzy(neutrosophic) twin vertices is $n$. Then $G$ is fixed-edge fuzzy(neutrosophic) graph.

Proof. Suppose $f$ and $f^{\prime}$ are two given edges. By supposition, every couple of vertices are fuzzy(neutrosophic) twin vertices. It implies $\mu(f)=\mu\left(f^{\prime}\right) . f$ and $f^{\prime}$ are arbitrary so every couple of edges have same values. It induces $G$ is fixed-edge fuzzy(neutrosophic) graph.

Corollary 4.4. Let $G$ be a fixed-vertex fuzzy(neutrosophic) graph. The number of fuzzy(neutrosophic) twin vertices is $n-1$. Then fuzzy(neutrosophic)-metric number is $n-2$, fuzzy(neutrosophic)-metric dimension is $(n-2) \sigma(m)$ where $m$ is fuzzy(neutrosophic) twin vertex with a vertex. Every $(n-2)$-set including fuzzy(neutrosophic) twin vertices is fuzzy(neutrosophic)-metric set.

Proof. By Corollary (4.2), fuzzy(neutrosophic)-metric number is $n-2$. By $G$ is a fixed-vertex fuzzy(neutrosophic) graph, fuzzy metric dimension is $(n-2) \sigma(m)$ where $m$ is fuzzy(neutrosophic) twin vertex with a vertex. One vertex doesn't belong to set of fuzzy(neutrosophic) twin vertices and a vertex from that set, are out of fuzzy metric set. It induces every $(n-2)$-set including fuzzy(neutrosophic) twin vertices is fuzzy metric set.

Proposition 4.5. Let $G$ be a fixed-vertex fuzzy(neutrosophic) graph such that it's fuzzy(neutrosophic) complete. Then fuzzy(neutrosophic)-metric number is $n-1$, fuzzy(neutrosophic)-metric dimension is $(n-1) \sigma(m)$ where $m$ is a given vertex. Every $(n-1)$-set is fuzzy(neutrosophic)-metric set.

Proof. In fuzzy(neutrosophic) complete, every couple of vertices are twin vertices. By $G$ is a fixed-vertex fuzzy(neutrosophic) graph and it's fuzzy(neutrosophic) complete, every couple of vertices are fuzzy(neutrosophic) twin vertices. Thus by Proposition (4.1), the result follows.

Proposition 4.6. Let $\mathcal{G}$ be a family of fuzzy(neutrosophic) graphs with common vertex set. Then simultaneously fuzzy(neutrosophic)-metric number of $\mathcal{G}$ is $n-1$.

Proof. Consider $(n-1)$-set. Thus there's no couple of vertices to be fuzzy(neutrosophic)-resolved. Therefore, every $(n-1)$-set is fuzzy(neutrosophic)-resolving set for any given fuzzy(neutrosophic) graph. Then it holds for any fuzzy(neutrosophic) graph. It implies it's fuzzy(neutrosophic)-resolving set and its cardinality is fuzzy(neutrosophic)-metric number. $(n-1)$-set has the cardinality $n-1$. Then it holds for any fuzzy(neutrosophic) graph. It induces it's simultaneously fuzzy(neutrosophic)-resolving set and its cardinality is simultaneously fuzzy(neutrosophic)-metric number. 
Proposition 4.7. Let $\mathcal{G}$ be a family of fuzzy(neutrosophic) graphs with common vertex set. Then simultaneously fuzzy(neutrosophic)-metric number of $\mathcal{G}$ is greater than the maximum fuzzy(neutrosophic)-metric number of $G \in \mathcal{G}$.

Proof. Suppose $t$ and $t^{\prime}$ are simultaneously fuzzy(neutrosophic)-metric number of $\mathcal{G}$ and fuzzy(neutrosophic)-metric number of $G \in \mathcal{G}$. Thus $t$ is fuzzy(neutrosophic)-metric number for any $G \in \mathcal{G}$. Hence, $t \geq t^{\prime}$. So simultaneously fuzzy(neutrosophic)-metric number of $\mathcal{G}$ is greater than the maximum fuzzy(neutrosophic)-metric number of $G \in \mathcal{G}$.

Proposition 4.8. Let $\mathcal{G}$ be a family of fuzzy(neutrosophic) graphs with common vertex set. Then simultaneously fuzzy(neutrosophic)-metric number of $\mathcal{G}$ is greater than simultaneously fuzzy(neutrosophic)-metric number of $\mathcal{H} \subseteq \mathcal{G}$.

Proof. Suppose $t$ and $t^{\prime}$ are simultaneously fuzzy(neutrosophic)-metric number of $\mathcal{G}$ and $\mathcal{H}$. Thus $t$ is fuzzy(neutrosophic)-metric number for any $G \in \mathcal{G}$. It implies $t$ is fuzzy(neutrosophic)-metric number for any $G \in \mathcal{H}$. So $t$ is simultaneously fuzzy(neutrosophic)-metric number of $\mathcal{H}$. By applying Definition about being the minimum number, $t \geq t^{\prime}$. So simultaneously fuzzy(neutrosophic)-metric number of $\mathcal{G}$ is greater than simultaneously fuzzy(neutrosophic)-metric number of $\mathcal{H} \subseteq \mathcal{G}$.

Theorem 4.9. Fuzzy(neutrosophic) twin vertices aren't fuzzy(neutrosophic)-resolved in any given fuzzy(neutrosophic) graph.

Proof. Let $t$ and $t^{\prime}$ be fuzzy(neutrosophic) twin vertices. Then $N(t)=N\left(t^{\prime}\right)$ and $\mu(t s)=\mu\left(t^{\prime} s\right)$, for all $s, s^{\prime} \in V$ such that $t s, t^{\prime} s \in E$. Thus for every given vertex $s^{\prime} \in V, d_{G}\left(s^{\prime}, t\right)=d_{G}(s, t)$ where $G$ is a given fuzzy(neutrosophic) graph. It means that $t$ and $t^{\prime}$ aren't resolved in any given fuzzy(neutrosophic) graph. $t$ and $t^{\prime}$ are arbitrary so fuzzy(neutrosophic) twin vertices aren't resolved in any given fuzzy(neutrosophic) graph.

Proposition 4.10. Let $G$ be a fixed-vertex fuzzy(neutrosophic) graph. If $G$ is fuzzy(neutrosophic) complete, then every couple of vertices are fuzzy(neutrosophic) twin vertices.

Proof. Let $t$ and $t^{\prime}$ be couple of given vertices. By $G$ is fuzzy(neutrosophic) complete, $N(t)=N\left(t^{\prime}\right)$. By $G$ is a fixed-vertex fuzzy(neutrosophic) graph, $\mu(t s)=\mu\left(t^{\prime} s\right)$, for all edges $t s, t^{\prime} s \in E$. Thus $t$ and $t^{\prime}$ are fuzzy(neutrosophic) twin vertices. $t$ and $t^{\prime}$ are arbitrary couple of vertices, hence every couple of vertices are fuzzy(neutrosophic) twin vertices.

Theorem 4.11. Let $\mathcal{G}$ be a family of fuzzy(neutrosophic) graphs with common vertex set and $G \in \mathcal{G}$ is a fixed-vertex fuzzy(neutrosophic) graph such that it's

fuzzy(neutrosophic) complete. Then simultaneously fuzzy(neutrosophic)-metric number is $n-1$, simultaneously fuzzy(neutrosophic)-metric dimension is $(n-1) \sigma(m)$ where $m$ is a given vertex. Every $(n-1)$-set is simultaneously fuzzy(neutrosophic)-metric set for $\mathcal{G}$.

Proof. $G$ is fixed-vertex fuzzy(neutrosophic) graph and it's fuzzy(neutrosophic) complete. So by Theorem (4.10), I get every couple of vertices in fuzzy(neutrosophic) complete are fuzzy(neutrosophic) twin vertices. So every couple of vertices, by Theorem (4.9), aren't resolved.

Corollary 4.12. Let $\mathcal{G}$ be a family of fuzzy(neutrosophic) graphs with fuzzy(neutrosophic) common vertex set and $G \in \mathcal{G}$ is a fuzzy(neutrosophic) complete. Then simultaneously fuzzy(neutrosophic)-metric number is $n-1$, simultaneously fuzzy(neutrosophic)-metric dimension is $(n-1) \sigma(m)$ where $m$ is a given vertex. Every $(n-1)$-set is simultaneously fuzzy(neutrosophic)-metric set for $\mathcal{G}$. 
Proof. By fuzzy(neutrosophic) graphs with fuzzy(neutrosophic) common vertex set, $G$ is fixed-vertex fuzzy(neutrosophic) graph. It's fuzzy(neutrosophic) complete. So by Theorem (4.11), I get intended result.

Theorem 4.13. Let $\mathcal{G}$ be a family of fuzzy(neutrosophic) graphs with common vertex set and for every given couple of vertices, there's a $G \in \mathcal{G}$ such that in that, they're fuzzy(neutrosophic) twin vertices. Then simultaneously fuzzy(neutrosophic)-metric number is $n-1$, simultaneously fuzzy(neutrosophic)-metric dimension is $(n-1) \sigma(m)$ where $m$ is a given vertex. Every $(n-1)$-set is simultaneously fuzzy(neutrosophic)-metric set for $\mathcal{G}$.

Proof. By Proposition (4.6), simultaneously fuzzy(neutrosophic)-metric number is $n-1$. By Theorem (4.9), simultaneously fuzzy(neutrosophic)-metric dimension is $(n-1) \sigma(m)$ where $m$ is a given vertex. Also, every $(n-1)$-set is simultaneously fuzzy(neutrosophic)-metric set for $\mathcal{G}$.

Theorem 4.14. Let $\mathcal{G}$ be a family of fuzzy(neutrosophic) graphs with common vertex set. If $\mathcal{G}$ contains three fixed-vertex fuzzy(neutrosophic) stars with different center, then simultaneously fuzzy(neutrosophic)-metric number is $n-2$, simultaneously fuzzy(neutrosophic)-metric dimension is $(n-2) \sigma(m)$ where $m$ is a given vertex. Every $(n-2)$-set is simultaneously fuzzy(neutrosophic)-metric set for $\mathcal{G}$.

Proof. The cardinality of set of fuzzy(neutrosophic) twin vertices is $n-1$. Thus by Corollary (4.4), the result follows.

Corollary 4.15. Let $\mathcal{G}$ be a family of fuzzy(neutrosophic) graphs with fuzzy(neutrosophic) common vertex set. If $\mathcal{G}$ contains three fuzzy(neutrosophic) stars with different center, then simultaneously fuzzy(neutrosophic)-metric number is $n-2$, simultaneously fuzzy(neutrosophic)-metric dimension is $(n-2) \sigma(m)$ where $m$ is a given vertex. Every $(n-2)$-set is simultaneously fuzzy(neutrosophic)-metric set for $\mathcal{G}$.

Proof. By fuzzy(neutrosophic) graphs with fuzzy(neutrosophic) common vertex set, $G$ is fixed-vertex fuzzy(neutrosophic) graph. It's fuzzy(neutrosophic) complete. So by Theorem (4.14), I get intended result.

\section{Antipodal Vertices}

\subsection{Even Fuzzy(Neutrosophic) Cycle}

Proposition 5.1. Consider two antipodal vertices $x$ and $y$ in any given fixed-edge even fuzzy(neutrosophic) cycle. Let $u$ and $v$ be given vertices. Then $d(x, u) \neq d(x, v)$ if and only if $d(y, u) \neq d(y, v)$.

Proof. $(\Rightarrow)$. Consider $d(x, u) \neq d(x, v)$. By

$d(x, u)+d(u, y)=d(x, y)=D(G), D(G)-d(x, u) \neq D(G)-d(x, v)$. It implies $d(y, u) \neq d(y, v)$.

$(\Leftarrow)$. Consider $d(y, u) \neq d(y, v)$. By

$d(y, u)+d(u, x)=d(x, y)=D(G), D(G)-d(y, u) \neq D(G)-d(y, v)$. It implies $d(x, u) \neq d(x, v)$.

Proposition 5.2. Consider two antipodal vertices $x$ and $y$ in any given fixed-edge even fuzzy(neutrosophic) cycle. Let $u$ and $v$ be given vertices. Then $d(x, u)=d(x, v)$ if and only if $d(y, u)=d(y, v)$. 
Proof. $(\Rightarrow)$. Consider $d(x, u)=d(x, v)$. By

$d(x, u)+d(u, y)=d(x, y)=D(G), D(G)-d(x, u)=D(G)-d(x, v)$. It implies $d(y, u)=d(y, v)$.

$(\Leftarrow)$. Consider $d(y, u)=d(y, v)$. By

$d(y, u)+d(u, x)=d(x, y)=D(G), D(G)-d(y, u)=D(G)-d(y, v)$. It implies $d(x, u)=d(x, v)$.

Proposition 5.3. The set contains two antipodal vertices, isn't fuzzy(neutrosophic)-metric set in any given fixed-edge even fuzzy(neutrosophic) cycle.

Proof. Let $x$ and $y$ be two given antipodal vertices in any given even fuzzy(neutrosophic) cycle. By Proposition (5.1), $d(x, u) \neq d(x, v)$ if and only if $d(y, u) \neq d(y, v)$. It implies that if $x$ fuzzy(neutrosophic)-resolves a couple of vertices, then $y$ fuzzy(neutrosophic)-resolves them, too. Thus either $x$ is in fuzzy(neutrosophic)-metric set or $y$ is. It induces the set contains two antipodal vertices, isn't fuzzy(neutrosophic)-metric set in any given even fuzzy(neutrosophic) cycle.

Proposition 5.4. Consider two antipodal vertices $x$ and $y$ in any given fixed-edge even fuzzy(neutrosophic) cycle. $x$ fuzzy(neutrosophic)-resolves a given couple of vertices, $z$ and $z^{\prime}$, if and only if $y$ does.

Proof. $(\Rightarrow)$. $x$ fuzzy(neutrosophic)-resolves a given couple of vertices, $z$ and $z^{\prime}$, then $d(x, z) \neq d\left(x, z^{\prime}\right)$. By Proposition (5.1), $d(x, z) \neq d\left(x, z^{\prime}\right)$ if and only if $d(y, z) \neq d\left(y, z^{\prime}\right)$. Thus $y$ fuzzy(neutrosophic)-resolves a given couple of vertices $z$ and $z^{\prime}$.

$(\Leftarrow)$. $y$ fuzzy(neutrosophic)-resolves a given couple of vertices, $z$ and $z^{\prime}$, then $d(y, z) \neq d\left(y, z^{\prime}\right)$. By Proposition (5.1), $d(y, z) \neq d\left(y, z^{\prime}\right)$ if and only if $d(x, z) \neq d\left(x, z^{\prime}\right)$. Thus $x$ fuzzy(neutrosophic)-resolves a given couple of vertices $z$ and $z^{\prime}$.

Proposition 5.5. There are two antipodal vertices aren't fuzzy(neutrosophic)-resolved by other two antipodal vertices in any given fixed-edge even fuzzy(neutrosophic) cycle.

Proof. Suppose $x$ and $y$ are a couple of vertices. It implies $d(x, y)=D(G)$. Consider $u$ and $v$ are another couple of vertices such that $d(x, u)=\frac{D(G)}{2}$. It implies $d(y, u)=\frac{D(G)}{2}$. Thus $d(x, u)=d(y, u)$. Therefore, $u$ doesn't fuzzy(neutrosophic)-resolve a given couple of vertices $x$ and $y$. By $D(G)=d(u, v)=d(u, x)+d(x, v)=\frac{D(G)}{2}+d(x, v)$, $d(x, v)=\frac{D(G)}{2}$. It implies $d(y, v)=\frac{D(G)}{2}$. Thus $d(x, v)=d(y, v)$. Therefore, $v$ doesn't fuzzy(neutrosophic)-resolve a given couple of vertices $x$ and $y$.

Proposition 5.6. For any two antipodal vertices in any given fixed-edge even fuzzy(neutrosophic) cycle, there are only two antipodal vertices don't fuzzy(neutrosophic)-resolve them

Proof. Suppose $x$ and $y$ are a couple of vertices such that they're antipodal vertices. Let $u$ be a vertex such that $d(x, u)=\frac{D(G)}{2}$. It implies $d(y, u)=\frac{D(G)}{2}$. Thus $d(x, u)=d(y, u)$. Therefore, $u$ doesn't fuzzy(neutrosophic)-resolve a given couple of vertices $x$ and $y$. Let $v$ be a antipodal vertex for $u$ such that $u$ and $v$ are antipodal vertices. Thus $v d(x, v)=\frac{D(G)}{2}$. It implies $d(y, v)=\frac{D(G)}{2}$. Therefore, $v$ doesn't fuzzy(neutrosophic)-resolve a given couple of vertices $x$ and $y$. If $u$ is a vertex such that $d(x, u) \neq \frac{D(G)}{2}$ and $v$ is a vertex such that $u$ and $v$ are antipodal vertices. Thus $d(x, v) \neq \frac{D(G)}{2}$ It induces either $d(x, u) \neq d(y, u)$ or $d(x, v) \neq d(y, v)$. It means either $u$ fuzzy(neutrosophic)-resolves a given couple of vertices $x$ and $y$ or $v$ fuzzy(neutrosophic)-resolves a given couple of vertices $x$ and $y$.

Proposition 5.7. In any given fixed-edge even fuzzy(neutrosophic) cycle, for any vertex, there's only one vertex such that they're antipodal vertices. 
Proof. If $d(x, y)=D(G)$, then $x$ and $y$ are antipodal vertices.

Proposition 5.8. Let $G$ be a fixed-edge even fuzzy(neutrosophic) cycle. Then every couple of vertices are fuzzy(neutrosophic)-resolving set if and only if they aren't antipodal vertices.

Proof. If $x$ and $y$ are antipodal vertices, then they don't fuzzy(neutrosophic)-resolve a given couple of vertices $u$ and $v$ such that they're antipodal vertices and $d(x, u)=\frac{D(G)}{2}$. Since $d(x, u)=d(x, v)=d(y, u)=d(y, v)=\frac{D(G)}{2}$.

Corollary 5.9. Let $G$ be a fixed-edge even fuzzy(neutrosophic) cycle. Then fuzzy(neutrosophic)-metric number is two.

Proof. A set contains one vertex $x$ isn't fuzzy(neutrosophic)-resolving set. Since it doesn't fuzzy(neutrosophic)-resolve a given couple of vertices $u$ and $v$ such that $d(x, u)=d(x, v)=1$. Thus fuzzy(neutrosophic)-metric number $\geq 2$. By Proposition (5.8), every couple of vertices such that they aren't antipodal vertices, are fuzzy(neutrosophic)-resolving set. Therefore, fuzzy(neutrosophic)-metric number is 2 .

Corollary 5.10. Let $G$ be a fixed-edge even fuzzy(neutrosophic) cycle. Then fuzzy(neutrosophic)-metric set contains couple of vertices such that they aren't antipodal vertices.

Proof. By Corollary (5.9), fuzzy(neutrosophic)-metric number is two. By Proposition (5.8), every couple of vertices such that they aren't antipodal vertices, are fuzzy(neutrosophic)-resolving set. Therefore, fuzzy(neutrosophic)-metric set contains couple of vertices such that they aren't antipodal vertices.

Corollary 5.11. Let $\mathcal{G}$ be a family of fixed-edge odd fuzzy(neutrosophic) cycles with fuzzy(neutrosophic) common vertex set. Then simultaneously fuzzy(neutrosophic)-metric set contains couple of vertices such that they aren't antipodal vertices and fuzzy(neutrosophic)-metric number is two.

\subsection{Odd Fuzzy(Neutrosophic) Cycle}

Proposition 5.12. In any given fixed-edge odd fuzzy(neutrosophic) cycle, for any vertex, there's no vertex such that they're antipodal vertices.

Proof. Let $G$ be a fixed-edge odd fuzzy(neutrosophic) cycle. if $x$ is a given vertex. Then there are two vertices $u$ and $v$ such that $d(x, u)=d(x, v)=D(G)$. It implies they aren't antipodal vertices.

Proposition 5.13. Let $G$ be a fixed-edge odd fuzzy(neutrosophic) cycle. Then every couple of vertices are fuzzy(neutrosophic)-resolving set.

Proof. Let $l$ and $l^{\prime}$ be couple of vertices. Thus, by Proposition (5.12), $l$ and $l^{\prime}$ aren't antipodal vertices. It implies for every given couple of vertices $f_{i}$ and $f_{j}$, I get either $d\left(l, f_{i}\right) \neq d\left(l, f_{j}\right)$ or $d\left(l^{\prime}, f_{i}\right) \neq d\left(l^{\prime}, f_{j}\right)$. Therefore, $f_{i}$ and $f_{j}$ are

fuzzy(neutrosophic)-resolved by either $l$ or $l^{\prime}$. It induces the set $\left\{l, l^{\prime}\right\}$ is fuzzy(neutrosophic)-resolving set.

Proposition 5.14. Let $G$ be a fixed-edge odd fuzzy(neutrosophic) cycle. Then fuzzy(neutrosophic)-metric number is two. 
Proof. Let $l$ and $l^{\prime}$ be couple of vertices. Thus, by Proposition (5.12), $l$ and $l^{\prime}$ aren't antipodal vertices. It implies for every given couple of vertices $f_{i}$ and $f_{j}$, I get either $d\left(l, f_{i}\right) \neq d\left(l, f_{j}\right)$ or $d\left(l^{\prime}, f_{i}\right) \neq d\left(l^{\prime}, f_{j}\right)$. Therefore, $f_{i}$ and $f_{j}$ are fuzzy(neutrosophic)-resolved by either $l$ or $l^{\prime}$. It induces the set $\left\{l, l^{\prime}\right\}$ is fuzzy(neutrosophic)-resolving set.

Corollary 5.15. Let $G$ be a fixed-edge odd fuzzy(neutrosophic) cycle. Then fuzzy(neutrosophic)-metric set contains couple of vertices.

Proof. By Proposition (5.14), fuzzy(neutrosophic)-metric number is two. By Proposition (5.13), every couple of vertices are fuzzy(neutrosophic)-resolving set. Therefore, fuzzy(neutrosophic)-metric set contains couple of vertices.

Corollary 5.16. Let $\mathcal{G}$ be a family of fixed-edge odd fuzzy(neutrosophic) cycles with fuzzy(neutrosophic) common vertex set. Then simultaneously fuzzy(neutrosophic)-metric set contains couple of vertices and fuzzy(neutrosophic)-metric number is two.

\section{Extended Results}

Proposition 6.1. If I use fixed-vertex strong fuzzy(neutrosophic) cycles instead of fixed-edge fuzzy(neutrosophic) cycles, then all results of Section (5) hold.

Proof. Let $G$ be a fixed-vertex strong fuzzy(neutrosophic) cycles. By $G$ is fuzzy(neutrosophic) strong and it's fixed-vertex, $G$ is fixed-edge fuzzy(neutrosophic).

Proposition 6.2. Let $G$ be a fixed-vertex strong fuzzy(neutrosophic) path. Then an 1-set contains leaf, is fuzzy(neutrosophic)-resolving set. An 1-set contains leaf, is fuzzy(neutrosophic)-metric set. Fuzzy(neutrosophic)-metric number is one.

Fuzzy(neutrosophic)-metric dimension is $\sigma(m)$ where $m$ is a given vertex.

Corollary 6.3. Let $\mathcal{G}$ be a family of fuzzy(neutrosophic) paths with common vertex set such that they've a common leaf. Then simultaneously fuzzy(neutrosophic)-metric number is 1 , simultaneously fuzzy(neutrosophic)-metric dimension is $\sigma(m)$ where $m$ is a given vertex. 1-set contains common leaf, is simultaneously fuzzy(neutrosophic)-metric set for $\mathcal{G}$.

Proposition 6.4. Let $G$ be a fixed-vertex strong fuzzy(neutrosophic) path. Then an 2-set contains every couple of vertices, is fuzzy(neutrosophic)-resolving set. An 2-set contains every couple of vertices, is fuzzy(neutrosophic)-metric set.

Fuzzy(neutrosophic)-metric number is two. Fuzzy(neutrosophic)-metric dimension is $2 \sigma(m)$ where $m$ is a given vertex.

Corollary 6.5. Let $\mathcal{G}$ be a family of fuzzy(neutrosophic) paths with common vertex set such that they've no common leaf. Then an 2-set is simultaneously fuzzy(neutrosophic)-resolving set, simultaneously fuzzy(neutrosophic)-metric number is 2 , simultaneously fuzzy(neutrosophic)-metric dimension is $2 \sigma(m)$ where $m$ is given vertices. Every 2-set is simultaneously fuzzy(neutrosophic)-metric set for $\mathcal{G}$.

Proposition 6.6. Let $G$ be a fixed-edge fuzzy(neutrosophic) t-partite. Then every set contains couple of vertices in different parts, is fuzzy(neutrosophic)-resolving set.

Corollary 6.7. Let $G$ be a fixed-vertex strong fuzzy(neutrosophic) t-partite. Then every $(n-2)$-set excludes two vertices from different parts, is fuzzy(neutrosophic)-resolving set. Every $(n-2)$-set excludes two vertices from different parts, is fuzzy(neutrosophic)-metric set. Fuzzy(neutrosophic)-metric number is $n-2$. Fuzzy(neutrosophic)-metric dimension is $(n-2) \sigma(m)$ where $m$ is a given vertex. 
Corollary 6.8. Let $G$ be a fixed-vertex strong fuzzy(neutrosophic) bipartite. Then every $(n-2)$-set excludes two vertices from different parts, is fuzzy(neutrosophic)-resolving set. Every $(n-2)$-set excludes two vertices from different parts, is fuzzy(neutrosophic)-metric set. Fuzzy(neutrosophic)-metric number is $n-2$. Fuzzy(neutrosophic)-metric dimension is $(n-2) \sigma(m)$ where $m$ is a given vertex.

Corollary 6.9. Let $G$ be a fixed-vertex strong fuzzy(neutrosophic) star. Then every $(n-2)$-set excludes center and a given vertex, is fuzzy(neutrosophic)-resolving set. An $(n-2)$-set excludes center and a given vertex, is fuzzy(neutrosophic)-metric set.

Fuzzy(neutrosophic)-metric number is $(n-2)$. Fuzzy(neutrosophic)-metric dimension is $(n-2) \sigma(m)$ where $m$ is a given vertex.

Corollary 6.10. Let $G$ be a fixed-vertex strong fuzzy(neutrosophic) wheel. Then every $(n-2)$-set excludes center and a given vertex, is fuzzy(neutrosophic)-resolving set. Every $(n-2)$-set excludes center and a given vertex, is fuzzy(neutrosophic)-metric set. Fuzzy(neutrosophic)-metric number is n-2. Fuzzy(neutrosophic)-metric dimension is $(n-2) \sigma(m)$ where $m$ is a given vertex.

Corollary 6.11. Let $\mathcal{G}$ be a family of fixed-vertex strong fuzzy(neutrosophic) t-partite with common vertex set. Then simultaneously fuzzy(neutrosophic)-metric number is $n-2$, simultaneously fuzzy(neutrosophic)-metric dimension is $(n-2) \sigma(m)$ Every $(n-2)$-set excludes two vertices from different parts, is simultaneously fuzzy(neutrosophic)-resolving set for $\mathcal{G}$. There's an $(n-2)$-set which is simultaneously fuzzy(neutrosophic)-metric set for $\mathcal{G}$.

Corollary 6.12. Let $\mathcal{G}$ be a family of fixed-vertex strong fuzzy(neutrosophic) bipartite with common vertex set. Then simultaneously fuzzy(neutrosophic)-metric number is $n-2$, simultaneously fuzzy(neutrosophic)-metric dimension is $(n-2) \sigma(m)$ Every $(n-2)$-set excludes two vertices from different parts, is simultaneously fuzzy(neutrosophic)-resolving set for $\mathcal{G}$. There's an $(n-2)$-set which is simultaneously fuzzy(neutrosophic)-metric set for $\mathcal{G}$.

Corollary 6.13. Let $\mathcal{G}$ be a family of fixed-vertex strong fuzzy(neutrosophic) star with common vertex set. Then simultaneously fuzzy(neutrosophic)-metric number is $n-2$, simultaneously fuzzy(neutrosophic)-metric dimension is $(n-2) \sigma(m)$ Every $(n-2)$-set excludes center and a given vertex, is simultaneously fuzzy(neutrosophic)-resolving set for $\mathcal{G}$. There's an $(n-2)$-set which is simultaneously fuzzy(neutrosophic)-metric set for $\mathcal{G}$.

Corollary 6.14. Let $\mathcal{G}$ be a family of fixed-vertex strong fuzzy(neutrosophic) wheel with common vertex set. Then simultaneously fuzzy(neutrosophic)-metric number is $n-2$, simultaneously fuzzy(neutrosophic)-metric dimension is $(n-2) \sigma(m)$ Every $(n-2)$-set excludes center and a given vertex, is simultaneously fuzzy(neutrosophic)-resolving set for $\mathcal{G}$. There's an $(n-2)$-set which is simultaneously fuzzy(neutrosophic)-metric set for $\mathcal{G}$.

\section{Applications}

Two applications are posed as follow.

\subsection{Located Places}

A program is devised for a robot to locate every couple of given places, separately. The number which this program assigns to any place from a given couple of places are unique. Thus every place has a unique number when a couple of places are given.

\section{7}




\subsection{Covid-19 and Identifying Infected People}

Dark network is description for infected people who are anonymous in the matter of Covid-19. Virus and its anonymously style to transmit the virus from one person to another person, could make a dark network involving people. Consider everyone as network titled fuzzy(neutrosophic). It means that the person and his networks containing his connections make two models, fixed-edge fuzzy(neutrosophic) and fixed-vertex strong fuzzy(neutrosophic). Now, I have a family of people which everyone is a model in the terms of Covid-19.

\section{Open Problems}

The crisp notion of dimension is defined on fuzzy(neutrosophic) graphs. Thus

Question 8.1. Is it possible to define fuzzy(neutrosophic) notion of dimension on fuzzy(neutrosophic) graphs?

There are too many limitations on the classes of fuzzy(neutrosophic) graphs by using fixed-edge fuzzy(neutrosophic) graphs and fixed-vertex strong fuzzy(neutrosophic) graphs.

Question 8.2. Is an approach existed to compute current dimension for specific classes of fuzzy(neutrosophic) graphs?

Question 8.3. What are basic attributes of current dimension for general classes of fuzzy(neutrosophic) graphs?

Finding other classes of fuzzy(neutrosophic) graphs has an ordinary approach to develop this study.

Question 8.4. Which new classes of fuzzy(neutrosophic) graphs are existed to develop this notion of current dimension?

Question 8.5. Which new classes of fuzzy(neutrosophic) graphs are existed to compute this notion of current dimension?

Question 8.6. Which general approaches are existed to study this notion of current dimension in fuzzy(neutrosophic) graphs?

Question 8.7. Which specific approaches are existed to study this notion of current dimension in fuzzy(neutrosophic) graphs?

Problem 8.8. Are there special crisp sets of vertices, e.g. antipodal vertices for fuzzy(neutrosophic) cycles, which have key role to study this notion of current dimension in fuzzy(neutrosophic) graphs?

Problem 8.9. Are there fuzzy(neutrosophic) special sets of vertices, e.g. fuzzy(neutrosophic) twin vertices for general classes, which have key role to study this notion of current dimension in fuzzy(neutrosophic) graphs?

\section{Discussion}

\section{Conclusion and Closing Remarks}

This study uses mixed combinations of fuzzy concepts and crisp concepts to explore new notion of crisp dimension in fuzzy(neutrosophic) graphs as individual and as family. In 
this way, some crisp notions like antipodal vertices are defined to use as a tool to study fuzzy(neutrosophic) cycles as individual and as family. Also, some fuzzy(neutrosophic) notions like fuzzy(neutrosophic) twin vertices are defined to use as a tool to study general classes of fuzzy(neutrosophic) graphs as individual and as family. Mixed family of fuzzy(neutrosophic) graphs are slightly studied by using fuzzy(neutrosophic) twin vertices and other ideas as individual and as family. In Table (5), I mention some advantages and limitations concerning this article and its proposed notions.

Table 5. A Brief Overview about Advantages and Limitations of this study

\begin{tabular}{|c|c|}
\hline Advantages & Limitations \\
\hline $\begin{array}{l}\text { 1. Using crisp and fuzzy(neutrosophic) } \\
\text { notions in one framework } \\
\text { together simultaneously. } \\
\text { 2. Study on fuzzy(neutrosophic) } \\
\text { as individual and as family. } \\
\text { 3. Involved classes as complete, } \\
\text { strong, path, cycle, t-partite, } \\
\text { bipartite, star, wheel. } \\
\text { 4. Characterizing classes of } \\
\text { fuzzy(neutrosophic) graphs } \\
\text { with smallest metric number } \\
\text { and largest metric number. }\end{array}$ & $\begin{array}{l}\text { 1. The most usages of fixed-edge } \\
\text { fuzzy(neutrosophic) graphs } \\
\text { and fixed-vertex strong } \\
\text { fuzzy(neutrosophic) graphs. }\end{array}$ \\
\hline
\end{tabular}

\section{References}

1. M. Akram, and G. Shahzadi, Operations on Single-Valued Neutrosophic Graphs, Journal of uncertain systems 11 (1) (2017) 1-26.

2. K. Atanassov, Intuitionistic fuzzy sets, Fuzzy Sets Syst. 20 (1986) 87-96.

3. S. Broumi, M. Talea, A. Bakali and F. Smarandache, Single-valued neutrosophic graphs, Journal of New Theory 10 (2016) 86-101.

4. N. Shah, and A. Hussain, Neutrosophic soft graphs, Neutrosophic Set and Systems 11 (2016) 31-44.

5. Henry Garrett, Big Sets Of Vertices, Preprints 2021, 2021060189 (doi: 10.20944/preprints202106.0189.v1).

6. Henry Garrett, Locating And Location Number, Preprints 2021, 2021060206 (doi: 10.20944/preprints202106.0206.v1).

7. Henry Garrett, Metric Dimensions Of Graphs, Preprints 2021, 2021060392 (doi: 10.20944/preprints202106.0392.v1).

8. Henry Garrett, New Graph Of Graph, Preprints 2021, 2021060323 (doi: 10.20944/preprints202106.0323.v1).

9. Henry Garrett, Numbers Based On Edges, Preprints 2021, 2021060315 (doi: 10.20944/preprints202106.0315.v1).

10. Henry Garrett, Matroid And Its Outlines, Preprints 2021, 2021060146 (doi: 10.20944/preprints202106.0146.v1). 
11. Henry Garrett, Matroid And Its Relations, Preprints 2021, 2021060080 (doi: 10.20944/preprints202106.0080.v1).

12. A. Shannon and K.T. Atanassov, A first step to a theory of the intuitionistic fuzzy graphs, Proceeding of FUBEST (Lakov, D., Ed.) Sofia (1994) 59-61.

13. F. Smarandache, A Unifying field in logics neutrosophy: Neutrosophic probability, set and logic, Rehoboth: American Research Press (1998).

14. H. Wang, F. Smarandache, Y. Zhang, and R. Sunderraman, Single-valued neutrosophic sets, Multispace and Multistructure 4 (2010) 410-413.

15. L. A. Zadeh, Fuzzy sets, Information and Control 8 (1965) 338-353. 